\title{
On four numerical schemes for a unipolar degenerate drift-diffusion model
}

Clément Cancès, Claire Chainais-Hillairet, Jürgen Fuhrmann and Benoît Gaudeul

\begin{abstract}
We consider a unipolar degenerate drift-diffusion system where the relation between the concentration of the charged species $c$ and the chemical potential $h$ is $h(c)=\log \frac{c}{1-c}$. For four different finite volume schemes based on four different formulations of the fluxes of the problem, we discuss stability and existence results. For two of them, we report a convergence proof. Numerical experiments illustrate the behaviour of the different schemes.
\end{abstract}

Key words: Finite Volume Methods, Drift-Diffusion Problems, Energy Methods

MSC (2010): 65M08, 65N08, 65Z05

\section{Introduction}

The transport of a charged species with density $c$ in the presence of a fixed or moving countercharge and a self-consistent electric field, deriving from an electrostatic potential $\Phi$, can be described by the non-dimensionalized system of equations

$$
\begin{aligned}
\partial_{t} c+\operatorname{div}(\mathbf{J})=0 & \text { in }(0, T) \times \Omega, \\
\mathbf{J}=-c \nabla(h(c)+\Phi) & \text { in }(0, T) \times \Omega,
\end{aligned}
$$

\footnotetext{
C. Cancès

Inria, Univ. Lille, CNRS, UMR 8524 - Laboratoire Paul Painlevé, F-59000 Lille

e-mail: clement.cances@inria.fr

C. Chainais-Hillairet \& B. Gaudeul

Univ. Lille, CNRS, UMR 8524, Inria - Laboratoire Paul Painlevé, F-59000 Lille

e-mail: \{claire.chainais, benoit.gaudeul\}@univ-lille.fr

J. Fuhrmann

Weierstrass Institute (WIAS), Mohrenstr. 39, 10117 Berlin, Germany

e-mail: juergen.fuhrmann@ wias-berlin.de
} 
where $h(c)=\log \left(\frac{c}{1-c}\right)$ is the chemical potential. The electrostatic potential $\Phi$ is related to space charge density via the Poisson equation

$$
-\Delta \Phi=c+c^{\text {dop }} \quad \text { in }(0, T) \times \Omega .
$$

In (3), $c^{\text {dop }}$ describes the doping profile of the media. Such models occur in applications ranging from organic semiconductors [5], high-temperature fuel cells [13] or simplified models of ionic liquids [8]. Because of the singularity of $h$ near 1, the density $c$ remains in the interval $(0,1)$. We consider the evolution in a connected bounded open domain $\Omega$ of $\mathbb{R}^{d}(d \leq 3)$ with polyhedral and Lipschitz continuous boundary $\partial \Omega$ during a finite but arbitrary time $T>0$. The doping profile $c^{\text {dop }}$ is assumed to be constant w.r.t. time and to be bounded, i.e., $c^{\text {dop }} \in L^{\infty}(\Omega)$. The system is supplemented with the prescription of the initial concentration

$$
c_{\left.\right|_{t=0}}=c^{0} \in L^{\infty}(\Omega) \quad \text { with } \quad 0 \leq c^{0} \leq 1 \quad \text { and } \quad 0<\bar{c}=\oint_{\Omega} c^{0} \mathrm{~d} \mathbf{x}<1,
$$

of no-flux boundary conditions for the concentration

$$
\mathbf{J} \cdot \mathbf{n}=0 \quad \text { on }(0, T) \times \partial \Omega .
$$

For the electrostatic potential, inhomogeneous Dirichlet boundary conditions are imposed on a subset $\Gamma_{D}$ of positive measure of $\partial \Omega$, whereas homogeneous Neumann boundary conditions are imposed on $\Gamma_{N}=\partial \Omega \backslash \Gamma_{D}$ :

$$
\Phi=\Phi^{D} \quad \text { on }(0, T) \times \Gamma_{D}, \quad \nabla \Phi \cdot \mathbf{n}=0 \quad \text { on }(0, T) \times \Gamma_{N} .
$$

We assume that $\Phi^{D}$ is defined in the whole domain, with $\Phi^{D} \in H^{1}(\Omega) \cap L^{\infty}(\Omega)$.

In [3], we studied and compared several Finite Volume schemes for the system (1)-(6). They are based on various reformulations of the flux $\mathbf{J}$ using the excess chemical potential $v(c)=h(c)-\log (c)=-\log (1-c)$, the activity and the inverse of the activity coefficient respectively defined by $a(c)=e^{h(c)}=\frac{c}{1-c}$, and $\beta(c)=\frac{c}{a(c)}=1-c$, or the diffusion enhancement $r(c)=-\log (1-c)$ satisfying $r^{\prime}(c)=c h^{\prime}(c)$. The flux $\mathbf{J}$, initially defined by (2), can be alternatively rewritten as

$$
\begin{aligned}
\mathbf{J} & =-\nabla c-c \nabla(\Phi+v(c)), \\
& =-\beta(c)(\nabla a(c)+a(c) \nabla \Phi), \\
& =-r^{\prime}(c) \nabla c-c \nabla \Phi .
\end{aligned}
$$

Let us notice that, even $v(c)=r(c)$ for our specific choice of $h(c)$, the excess chemical potential and the diffusion enhancement arising respectively in (7) and (9) have a different physical sense so that we keep different notations.

Each formulation (2), (7), (8) and (9) leads to a different scheme that we compared from a numerical analysis point of view. Notice that the flux $\mathbf{J}$ can also be expressed as $\mathbf{J}=-\nabla r(c)-c \nabla \Phi$. This last formulation is used todefine a proper notion 
of weak solution to (1)-(6). In order to state this definition, we introduce the vector space $\mathscr{H}_{\Gamma^{D}}=\left\{f \in H^{1}(\Omega), f_{\left.\right|_{D}}=0\right\}$ and the space-time cylinder $Q_{T}=(0, T) \times \Omega$.

Definition 1. A couple $(c, \Phi)$ is a weak solution of (1)-(6) if

- $c \in L^{\infty}\left(Q_{T} ;[0,1]\right) \quad$ with $\quad r(c) \in L^{2}\left((0, T) ; H^{1}(\Omega)\right)$, and $\Phi-\Phi^{D} \in$ $L^{\infty}\left((0, T), \mathscr{H}_{\Gamma^{D}}\right)$

- for all $\varphi \in C_{c}^{\infty}([0, T) \times \bar{\Omega})$, there holds

$$
\iint_{Q_{T}} c \partial_{t} \varphi \mathrm{d} \mathbf{x} \mathrm{d} t+\int_{\Omega} c^{0} \varphi(0, \cdot) \mathrm{d} \mathbf{x}-\iint_{Q_{T}}(\nabla r(c)+c \nabla \Phi) \cdot \nabla \varphi \mathrm{d} \mathbf{x} \mathrm{d} t=0 ;
$$

- for all $\psi \in \mathscr{H}_{\Gamma^{D}}$ and almost all $t \in(0, T)$, there holds

$$
\int_{\Omega} \nabla \Phi(t, \mathbf{x}) \cdot \nabla \psi(\mathbf{x}) \mathrm{d} \mathbf{x}=\int_{\Omega}\left(c(t, \mathbf{x})+c^{\mathrm{dop}}(\mathbf{x})\right) \psi(\mathbf{x}) \mathrm{d} \mathbf{x} .
$$

We shortly discuss the gradient flow structure of the system (1)-(6). Define the mixing entropy density

$$
H(c)=c \log (c)+(1-c) \log (1-c),
$$

which is an antiderivative of $h$, then the electrochemical energy is given by

$$
E(c, \Phi)=\int_{\Omega}\left\{H(c)+\frac{1}{2}|\nabla \Phi|^{2}\right\} \mathrm{d} \mathbf{x}-\int_{\Gamma_{D}} \Phi^{D} \nabla \Phi \cdot \mathbf{n} \mathrm{d} \gamma .
$$

The electrochemical energy is a Lyapunov functional. Moreover, the dissipation rate for the energy is explicitly given.

Proposition 1.1 Let $(c, \Phi)$ be a smooth solution to (1)-(6), with c bounded away from 0 and 1 , then

$$
\frac{\mathrm{d}}{\mathrm{d} t} E(c, \Phi)+\int_{\Omega} c|\nabla(h(c)+\Phi)|^{2} \mathrm{~d} \mathbf{x}=0 .
$$

\section{TPFA Finite Volume approximations}

For the space discretization, we use the standard notation of an admissible finite volume mesh $\left(\mathscr{T}, \mathscr{E},\left(\mathbf{x}_{K}\right)_{K \in \mathscr{T}}\right)$, see [3]. Control volumes are denoted by $K \in \mathscr{T}$ with respective measures $m_{K}$, whereas edges are denoted by $\sigma \in \mathscr{E}$, their $(d-1)$ dimensional measure being denoted by $m_{\sigma}$. Since our method relies on a two-point flux approximation, we suppose that the mesh satisfies the classical orthogonality condition [6, Chapter 9]. For the time discretization, we consider an increasing finite family of times $0=t_{0}<t_{1}<\cdots<t_{N}=T$. We denote by $\Delta t_{n}=t_{n}-t_{n-1}$ for $1 \leq$ $n \leq N$, by $\Delta t=\left(\Delta t_{n}\right)_{1 \leq n \leq N}$, and by $\overline{\Delta t}=\max _{1 \leq n \leq N} \Delta t_{n}$. 
The initial data $c_{0}$ and the doping profile $c^{\text {dop }}$ are respectively discretized into $\left(c_{K}^{0}\right)_{K \in \mathscr{T}},\left(c_{K}^{\text {dop }}\right)_{K \in \mathscr{T}} \in \mathbb{R}^{\mathscr{T}}$ by setting

$$
c_{K}^{0}=\frac{1}{m_{K}} \int_{K} c^{0}(\mathbf{x}) \mathrm{d} \mathbf{x}, \quad c_{K}^{\mathrm{dop}}=\frac{1}{m_{K}} \int_{K} c^{\mathrm{dop}}(\mathbf{x}) \mathrm{d} \mathbf{x}, \quad \forall K \in \mathscr{T},
$$

Assume that $c^{n-1}=\left(c_{K}^{n-1}\right)_{K \in \mathscr{T}} \in[0,1]^{\mathscr{T}}$ is given for some $n>0$. We define how to compute $\left(c^{n}, \Phi^{n}\right)=\left(c_{K}^{n}, \Phi_{K}^{n}\right)_{K \in \mathscr{T}}$. For all $K \in \mathscr{T}$ and all $\sigma \in \mathscr{E}_{K}=\mathscr{E}_{\text {int }} \cup \mathscr{E}_{\text {ext }}$, the set of interior and exterior control volume facets, we define the mirror values $c_{K \sigma}^{n}$ and $\Phi_{K \sigma}^{n}$ of $c_{K}^{n}$ and $\Phi_{K}^{n}$ respectively across $\sigma$ by setting

$c_{K \sigma}^{n}=\left\{\begin{array}{lll}c_{L}^{n} & \text { if } \sigma=K \mid L \in \mathscr{E}_{\text {int }}, \\ c_{K}^{n} & \text { if } \sigma \in \mathscr{E}_{\mathrm{ext}},\end{array} \quad \Phi_{K \sigma}^{n}= \begin{cases}\Phi_{L}^{n} & \text { if } \sigma=K \mid L \in \mathscr{E}_{\text {int }}, \\ \Phi_{K}^{n} & \text { if } \sigma \in \mathscr{E}^{N}, \\ \Phi_{\sigma}^{n}=\frac{1}{m_{\sigma}} \int_{\sigma} \Phi^{D} \mathrm{~d} \gamma & \text { if } \sigma \in \mathscr{E}^{D} .\end{cases}\right.$

For $\sigma \in \mathscr{E}$, we set $d_{\sigma}=\left|\mathbf{x}_{K}-\mathbf{x}_{L}\right|$ if $\sigma=K\left|L \in \mathscr{E}_{\text {int }}, d_{\sigma}=\right| \mathbf{x}_{K}-\mathbf{x}_{\sigma} \mid$ if $\sigma \in \mathscr{E}_{\text {ext }}$, and $\tau_{\sigma}=\frac{m_{\sigma}}{d_{\sigma}}$. Given $u=\left(u_{K}\right)_{K \in \mathscr{T}} \in \mathbb{R}^{\mathscr{T}}$, we define the oriented and absolute jumps of $u$ across $\sigma \in \mathscr{E}_{K}$ by $D_{K \sigma} u=u_{K \sigma}-u_{K}$, and $D_{\sigma} u=\left|D_{K \sigma} u\right|$.

All the four schemes we consider are based on a backward Euler scheme for the time discretization and a TPFA finite volume scheme for the space discretization. They are written as follows:

$$
\begin{gathered}
-\sum_{\sigma \in \mathscr{E}_{K}} \tau_{\sigma} D_{K \sigma} \Phi^{n}=m_{K}\left(c_{K}^{n}+c_{K}^{\mathrm{dop}}\right), \quad \forall K \in \mathscr{T}, \\
m_{K} \frac{c_{K}^{n}-c_{K}^{n-1}}{\Delta t_{n}}+\sum_{\sigma \in \mathscr{E}_{K, \text { int }}} F_{K \sigma}^{n}=0, \quad \forall K \in \mathscr{T} .
\end{gathered}
$$

To close the system (14), it remains to define the numerical fluxes $F_{K \sigma}^{n}$. Due to the no-flux boundary condition, we only have to define the inner fluxes. They are defined with a function $\mathscr{F}$ of the primary unknowns $\left(c_{K}^{n}, c_{L}^{n}, \Phi_{K}^{n}, \Phi_{L}^{n}\right)$ :

$$
F_{K \sigma}^{n}=\tau_{\sigma} \mathscr{F}\left(c_{K}^{n}, c_{L}^{n}, \Phi_{K}^{n}, \Phi_{L}^{n}\right), \quad \forall K \in \mathscr{T}, \forall \sigma=K \mid L .
$$

The different schemes considered in this contribution correspond to different choices of $\mathscr{F}$. All of them verify $\mathscr{F}\left(c_{K}, c_{L}, \Phi_{K}, \Phi_{L}\right)=-\mathscr{F}\left(c_{L}, c_{K}, \Phi_{L}, \Phi_{K}\right)$, so that the numerical fluxes are locally conservative. Three of the four schemes are extensions of the Scharfetter-Gummel scheme [12] and feature the Bernoulli function $B(u)=\frac{u}{e^{u}-1}$.

The centred flux is derived from (2), which suggests the following definition:

$$
\mathscr{F}\left(c_{K}, c_{L}, \Phi_{K}, \Phi_{L}\right)=-\frac{c_{K}+c_{L}}{2} D_{K \sigma}(h(c)+\Phi) .
$$

The associate flux can be seen as a particular case in the TPFA context of the fluxes introduced in [4]. This scheme is not based on the Scharfetter-Gummel scheme. 
The Sedan flux is named after the SEDAN III simulator [14]. Formula (7) for the flux $\mathbf{J}$ suggests to use a classical Scharfetter-Gummel scheme, but for a modified potential $\Phi+v(c)$ instead of only $\Phi$, leading to

$$
\mathscr{F}\left(c_{K}, c_{L}, \Phi_{K}, \Phi_{L}\right)=B\left(D_{K \sigma}(\Phi+v(c))\right) c_{K}-B\left(-D_{K \sigma}(\Phi+v(c))\right) c_{L} .
$$

The activity based flux is a restriction of the flux introduced in [7]. It relies on the expression (8). With frozen $\beta(c)$, the flux $\mathbf{J}$ is linear w.r.t. $a(c)$. This suggests choosing a particular average for $\beta(c)$-here the arithmetic mean- and applying the Scharfetter-Gummel scheme to approximate $-\nabla a(c)-a(c) \nabla \Phi$, yielding

$$
\mathscr{F}\left(c_{K}, c_{L}, \Phi_{K}, \Phi_{L}\right)=\frac{\beta\left(c_{K}\right)+\beta\left(c_{L}\right)}{2}\left\{B\left(D_{K \sigma} \Phi\right) a\left(c_{K}\right)-B\left(-D_{K \sigma} \Phi\right) a\left(c_{L}\right)\right\} .
$$

Formula (9) for the flux $\mathbf{J}$ suggests that, with introducing a variable diffusion coefficient approximating the $r^{\prime}(c)$ per face, one can use the Scharfetter-Gummel scheme. Following [1], the approximation $\mathfrak{d} r\left(c_{K}, c_{L}\right)$ of $r^{\prime}(c)$ is defined as

$$
\mathfrak{d} r\left(c_{K}, c_{L}\right)= \begin{cases}\frac{h\left(c_{K}\right)-h\left(c_{L}\right)}{\log \left(c_{K}\right)-\log \left(c_{L}\right)} & \text { if } c_{K} \neq c_{L}, \\ r^{\prime}\left(c_{K}\right) & \text { if } c_{K}=c_{L} .\end{cases}
$$

This leads to the following definition of the Bessemoulin-Chatard flux [1]:

$$
\mathscr{F}\left(c_{K}, c_{L}, \Phi_{K}, \Phi_{L}\right)=\mathfrak{d} r\left(c_{K}, c_{L}\right)\left\{B\left(\frac{D_{K \sigma} \Phi}{\mathfrak{d} r\left(c_{K}, c_{L}\right)}\right) c_{K}-B\left(-\frac{D_{K \sigma} \Phi}{\mathfrak{d} r\left(c_{K}, c_{L}\right)}\right) c_{L}\right\} .
$$

\subsection{Main results}

The energy decay was one of the key properties of the continuous model, cf. Proposition 1.1. This property is transposed to the discrete setting by all the four discretizations we have considered. The discrete energy functional $E_{\mathscr{T}}$ has to be thought of as a discrete counterpart of the continuous energy functional $E$, cf. (12). It is defined by:

$$
E_{\mathscr{T}}\left(c^{n}, \Phi^{n}\right)=\sum_{K \in \mathscr{T}} m_{K} H\left(c_{K}^{n}\right)+\frac{1}{2} \sum_{\sigma \in \mathscr{E}} \tau_{\sigma}\left(D_{\sigma} \Phi^{n}\right)^{2}-\sum_{K \in \mathscr{T}} \sum_{\sigma \in \mathscr{E} D \cap \mathscr{E}_{K}} \tau_{\sigma} \Phi_{\sigma}^{D} D_{K \sigma} \Phi^{n} .
$$

Our first result focuses on the four schemes on a fixed mesh. It states that the nonlinear system corresponding to each scheme admits a solution which preserves the physical bounds on the concentrations and the decay of the energy:

Theorem 1. Let $\left(\mathscr{T}, \mathscr{E},\left(\mathbf{x}_{K}\right)_{K \in \mathscr{T}}\right)$ be an admissible mesh and let $c^{0}$ be defined by (13). Then, for all $1 \leq n \leq N$, the nonlinear system of equations (14)-(15), supple- 
mented either with $(\mathrm{C}),(\mathrm{S}),(\mathrm{AB})$, or $(\mathrm{BC})$, has a solution $\left(c^{n}, \Phi^{n}\right) \in[0,1]^{\mathscr{T}} \times \mathbb{R}^{\mathscr{T}}$. Moreover, the solution to the scheme satisfies, for all $1 \leq n \leq N$,

$$
E_{\mathscr{T}}\left(c^{n,}, \Phi^{n}\right) \leq E_{\mathscr{T}}\left(c^{n-1}, \Phi^{n-1}\right) \quad \text { and } \quad 0<c_{K}^{n}<1, \quad \forall K \in \mathscr{T}
$$

Once a discrete solution to the scheme $\left(c^{n}, \Phi^{n}\right)_{1 \leq n \leq N}$ at hand, we can define an approximate solution $\left(c_{\mathscr{T}, \Delta t}, \Phi_{\mathscr{T}, \Delta t}\right)$. It is the piecewise constant function defined almost everywhere by

$$
c_{\mathscr{T}, \Delta t}(t, \mathbf{x})=c_{K}^{n}, \quad \Phi_{\mathscr{T}, \Delta t}(t, \mathbf{x})=\Phi_{K}^{n} \quad \text { if }(t, \mathbf{x}) \in\left(t_{n-1}, t_{n}\right] \times K .
$$

Let $\left(\mathscr{T}_{m}, \mathscr{E}_{m},\left(\mathbf{x}_{K}\right)_{K \in \mathscr{T}_{m}}\right)_{m \geq 1}$ be a sequence of admissible meshes such that $h_{\mathscr{T}_{m}}, \overline{\Delta t}_{m} \underset{m \rightarrow \infty}{\longrightarrow} 0$ while the mesh regularity remains bounded (see [3] for the definition of the regularity of the mesh). A natural question is the convergence of the associated sequence of approximate solutions $\left(c_{\mathscr{T}_{m}, \Delta t_{m}}, \Phi_{\mathscr{T}_{m}, \Delta t_{m}}\right)_{m \geq 1}$ towards a weak solution to the continuous problem. The convergence result is stated in Theorem 2, only for the centred scheme and the Sedan scheme. The proof is detailed in [3]. It is based on compactness arguments. As far as we know, there is no uniqueness result for the weak solutions, hence the convergence only holds up to a subsequence.

Theorem 2. For the centred scheme (inner fluxes defined by (15) and (C)) and the Sedan scheme (inner fluxes defined by (15) and (S)), a sequence of approximate solutions $\left(c_{\mathscr{T}_{m}, \Delta t_{m}}, \Phi_{\mathscr{T}_{m}, \Delta t_{m}}\right)_{m \geq 1}$ satisfies, up to a subsequence,

$$
c_{\mathscr{T}_{m}, \Delta t_{m}} \underset{m \rightarrow \infty}{\longrightarrow} c \quad \text { a.e. in } Q_{T}, \quad \Phi_{\mathscr{T}_{m}, \Delta t_{m}} \underset{m \rightarrow \infty}{\longrightarrow} \Phi \quad \text { in } L^{2}\left(Q_{T}\right),
$$

where $(c, \Phi)$ is a weak solution to (1)-(6) in the sense of Definition 1.

\section{A numerical example}

The presented numerical example and those in [3] have been implemented in the Julia language [2] based on the packages VoronoifVM.jl [9] and ForwardDiff.jl[11].

The example is a modification of one of the numerical examples in [3]. It considers the problem (1)-(3) in $\Omega=(0,50)$ with homogeneous Dirichlet boundary conditions for $\Phi$ and homogeneous Neumann boundary conditions for $c$ with $c^{\mathrm{dop}}=-0.5$ and $c_{0}=0.7$. We choose a self-consistent initial value $\Phi_{0}$ for the electrostatic potential such that (3) is fulfilled for $c_{0}$.

For this test case, the four schemes behave similarly, as shown in the right picture of Fig. 1. As demonstrated in [3], more extreme examples forcing concentrations to be close to the physical bounds reveal important differences. The left picture of Fig.1 shows that, for large $t$, the charge carrier concentration approaches a steady state with two space charge regions. We remark that $c$ stays in the range $(0,1)$, and that the energy (12) decreases during the time evolution for all four schemes 

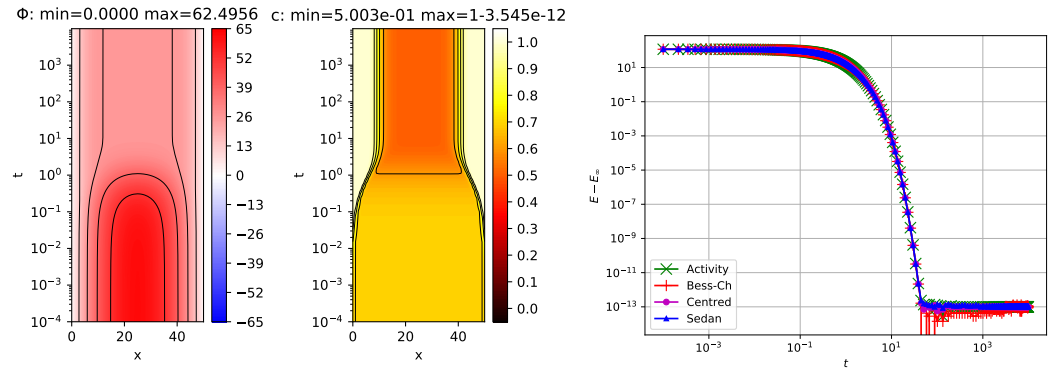

Fig. 1 Left: time evolution of solution for scheme (S) on domain $\Omega=(0,50)$ with constant initial value $c=0.7$, homogeneous Dirichlet boundary conditions for $\Phi, c^{\text {dop }}=-\frac{1}{2}$ and homogeneous Neumann boundary conditions for $c$. Right: Evolution of the relative free energy according to (12) for the different schemes.


Fig. 2 Error $e_{0}$ in $L^{2}\left((0, T) ; L^{2}(\Omega)^{2}\right)$ (left) and $e_{1}$ in $L^{2}\left((0, T) ; H^{1}(\Omega)^{2}\right)$ on $(c, \Phi)$ for scheme (S) vs. optimal energy dissipation per time step $\mathscr{D}_{\text {opt }}$ for grid step sizes $h=0.5 * 2^{-m}$ for $m=1 \ldots 6$.

discussed in this paper, as stated in Theorem 1. At the end of the time evolution, an electroneutral region occurs in the center of the domain. At both boundaries, equally charged space regions set up enrichment boundary layers due to the fact that the amount of charge carriers confined to the domain cannot be compensated by the doping.

For the convergence experiment (see Fig. 2) we present results for scheme (S) only, the other schemes discussed perform similarly. For the space discretization, we used 6 levels of refinement building on a subdivision into 100 intervals for the coarsest mesh. Following a suggestion of Gajewski and Gärtner [10], we used an adaptive strategy based on the equidistribution of the energy dissipated per time step for the control of the time step size. We start with $t_{1}=10^{-4}$ and use the following expression to calculate the next time step:

$$
t_{n+1}=\min \left\{t_{n} \cdot 1.2, t_{n} \cdot \frac{\mathscr{D}_{o p t}}{\mathscr{D}_{n}}, 100\right\},
$$


where $\mathscr{D}_{n}=\left|E^{n}-E^{n-1}\right|$ is the change in the free energy during the previous timestep and $\mathscr{D}_{\text {opt }}$ is the parameter which controls the time step size. This approach ensures that the energy dissipated per time step remains of the same order as $\mathscr{D}_{\text {opt }}$ outside of a start region where the time-step size is ramped up and a final region where the dissipation rate approaches zero.

In Fig. 2, we show for a sequence of meshes the convergence of the $L^{2}\left(L^{2}\right)$ and $L^{2}\left(H^{1}\right)$ errors for the approximate solution $\left(c_{\mathscr{T}, \Delta t}, \Phi_{\mathscr{T}, \Delta t}\right)$ with respect to a reference solution calculated on a fine space-time grid. For coarse space discretizations, errors are dominated by the spatial error, and decreasing the time step control parameter $\mathscr{D}_{\text {opt }}$ does not decrease the overall error. On the other hand, on fine spatial grids, we observe that the errors seem to decrease proportionally to the square root of $\mathscr{D}_{\text {opt }}$ which gives rise to a corresponding hypothesis to be investigated in further research.

\section{References}

1. M. Bessemoulin-Chatard. A finite volume scheme for convection-diffusion equations with nonlinear diffusion derived from the Scharfetter-Gummel scheme. Numer. Math., 121(4):637670, 2012.

2. J. Bezanson, Al. Edelman, S. Karpinski, and Viral B Shah. Julia: A fresh approach to numerical computing. SIAM review, 59(1):65-98, 2017.

3. C. Cancès, C. Chainais-Hillairet, J. Fuhrmann, and B. Gaudeul. A numerical analysis focused comparison of several finite volume schemes for a unipolar degenerate drift-diffusion model. https://hal.archives-ouvertes.fr/hal-02194604, to appear in IMA J. of Num. Anal., 2020

4. C. Cancès and C. Guichard. Numerical analysis of a robust free energy diminishing finite volume scheme for parabolic equations with gradient structure. Found. Comput. Math., 17(6):1525-1584, 2017.

5. R. Coehoorn, W.F. Pasveer, P.A. Bobbert, and M.A.J. Michels. Charge-carrier concentration dependence of the hopping mobility in organic materials with gaussian disorder. Physical Review B, 72(15):155206, 2005.

6. R. Eymard, T. Gallouët, and R. Herbin. Finite volume methods. Ciarlet, P. G. (ed.) et al., in Handbook of numerical analysis. North-Holland, Amsterdam, pp. 713-1020, 2000.

7. J. Fuhrmann. Comparison and numerical treatment of generalised NernstPlanck models. Comput. Phys. Commun., 196:166-178, 2015.

8. J. Fuhrmann. A numerical strategy for Nernst-Planck systems with solvation effect. Fuel Cells, 16, 122016.

9. J. Fuhrmann. VoronoiFVM.jl: Solver for coupled nonlinear partial differential equations based on the voronoi finite volume method, 2019. DOI:10.5281/zenodo.3529808.

10. H. Gajewski and K. Gärtner. On the discretization of van Roosbroeck's equations with magnetic field. Z. Angew. Math. Mech., 76(5):247-264, 1996.

11. J. Revels, M. Lubin, and T. Papamarkou. Forward-mode automatic differentiation in julia. arXiv:1607.07892 [cs.MS], 2016.

12. D. L. Scharfetter and H. K. Gummel. Large-signal analysis of a silicon read diode oscillator. Electron Devices, IEEE Transactions on, 16(1):64-77, 1969.

13. P. Vágner, C. Guhlke, V. Miloš, R. Müller, and J. Fuhrmann. A continuum model for yttriastabilized zirconia incorporating triple phase boundary, lattice structure and immobile oxide ions. Journal of Solid State Electrochemistry, pages 1-20, 2019.

14. Z. Yu and R. Dutton. SEDAN III. www-tcad.stanford.edu/tcad/programs/sedan3.html, 1988. 